\title{
The Significance of Early Pregnancy Units in the Practice of a B usy Obstetric Unit
}

\author{
${ }^{1}$ Mandy Abushama, ${ }^{2}$ Halima Almuhanadi \\ ${ }^{1}$ Consultant, Department of Obstetrics and Gynecology, Women's Hospital, Hamad Medical Corporation, Doha, Qatar \\ ${ }^{2} S$ pecialist, Department of Obstetrics and Gynecology, Women's Hospital, Hamad Medical Corporation, Doha, Qatar
}

Correspondence: Mandy Abushama, Consultant, Department of O bstetrics and G ynecology, Women's Hospital, Hamad Medical Corporation, PO Box 3050, Doha, Qatar, e-mail: mdabushama@ hotmail.com

\section{ABSTRACT}

A significant proportion of women experience bleeding in early pregnancy resulting in considerable distress. Early pregnancy unit (EPAU) should promote the sensitive and efficient management of women with early pregnancy problems and we believe this is best achieved by the establishment of a dedicated unit with dedicated staff. This paper outlines the experience of setting up an early pregnancy unit in a busy obstetrical unit.

Keywords: Miscarriage, Early pregnancy assessment, Obstetric unit.

\section{INTRODUCTION}

M iscarriage is the most common complication of pregnancy, affecting 12 to $15 \%$ of all recognized pregnancies and a substantially greater proportion (up to $40 \%$ ) of biochemical pregnancies. ${ }^{1}$ It is now recognized that a much greater proportion of women experience bleeding in the first trimester and this requires prompt diagnosis and treatment in order to alleviate anxiety. ${ }^{2}$ This large proportion of pregnant women were traditionally admitted at all times of day and night for observation followed the next day with an ultrasound examination. Discharge or operative management follows leading to a lack of satisfaction and a scattered service. ${ }^{3}$ The gold standard would be a timely effective and reassuring service that can be audited in the form of an early pregnancy unit (EPAU).

A $\mathrm{EPAU}$ should promote the sensitive and efficient management of women with early pregnancy problems and we believe this is best achieved by the establishment of a dedicated unit with dedicated staff.

Being a busy hospital with over 14000 deliveries a year, the administration saw fit to start a new unitfor early pregnancy assessment unit (EPAU). This was in line with the recommendation of the Royal College of Obstetricians to establish such units since 2006. ${ }^{4,5}$ In this paper, we wished to quantify the early pregnancy assessment service after a year of running.

We established our unit early in 2009 with a dedicated area and dedicated staff. The staff include two nurses and a special ist doctor managing the unit with the help of other trained physicians, as well as a clerical staff. The area is divided into a waiting area and an examination room. The unit runs five days a week, from 8 am to $3 \mathrm{pm}$. It receives patients from the emergency room, general practice and private clinics. The criteria for admission included a positive pregnancy test with amenorrhea up to 20 weeks.

\section{METHODS}

Patients attending the unit during the period betw een January and $M$ arch 2010 were seen by specialists trained by the fellowship program in obstetric and gynaecological ultrasound at Hamad Medical Corporation. They filled in a specially formulated questionnaire that was approved by the research and ethics committee about each visit. A total of 450 such forms were filled and the data were analyzed. A II cases were taken until the study number was completed.

\section{RESULTS}

The unit has got pretty busy during 2009 with an average of 340 patients a month, a third of which are reviews, i.e. repeat visits.

During our study period, the vast majority of the patients were A rabs, both Q atari and non-Q atari (Fig. 1). The large majority of patients were referred by the accident and emergency as detailed in Figure 2. Vaginal bleeding was by far the commonest presentation (Fig. 3). Seven percent had ever used contraception. A mong these, the intrauterine contraceptive device and the combined pill were by far the commonest contraceptive methods used (Fig. 4).

Sixteen patients had a previous ectopic (4\%) which is in line with international figures of countries with a relatively low sexually transmitted disease incidence.

\section{DISCUSSION}

B leeding in early pregnancy generates an enormous amount of anxiety for the patients. Prior to the introduction of the EPAU, women were admitted at all hours of the day and night by tired and junior doctors. They waited several hours in the hospital bed for a viability scan and fasted for prolonged periods prior 


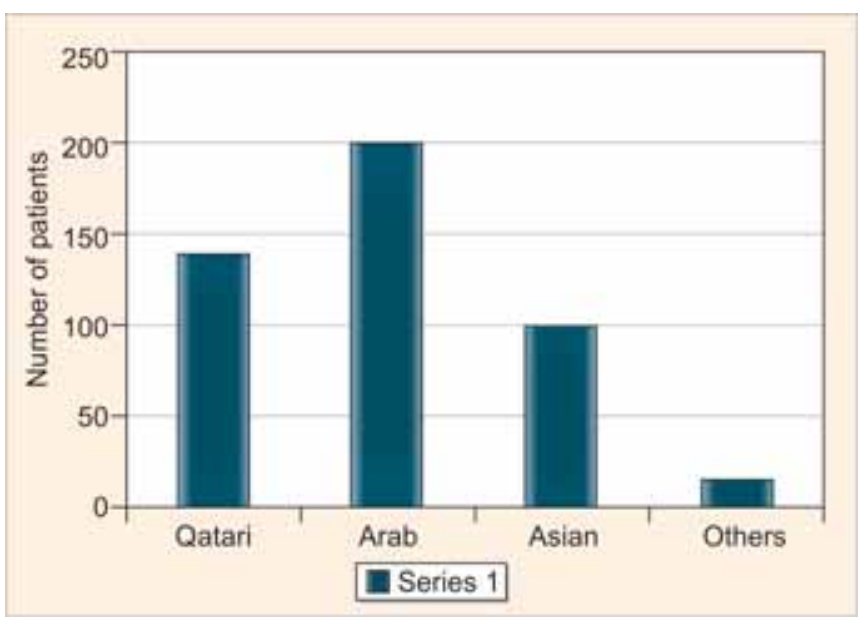

Fig. 1: The number of patients attending by nationality

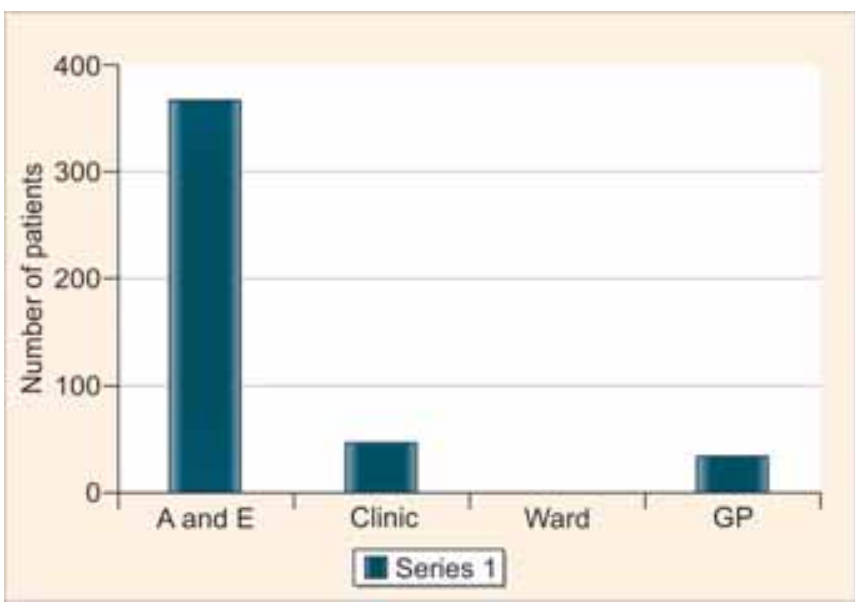

Fig. 2: The area from which the patients were referred to the EPAU

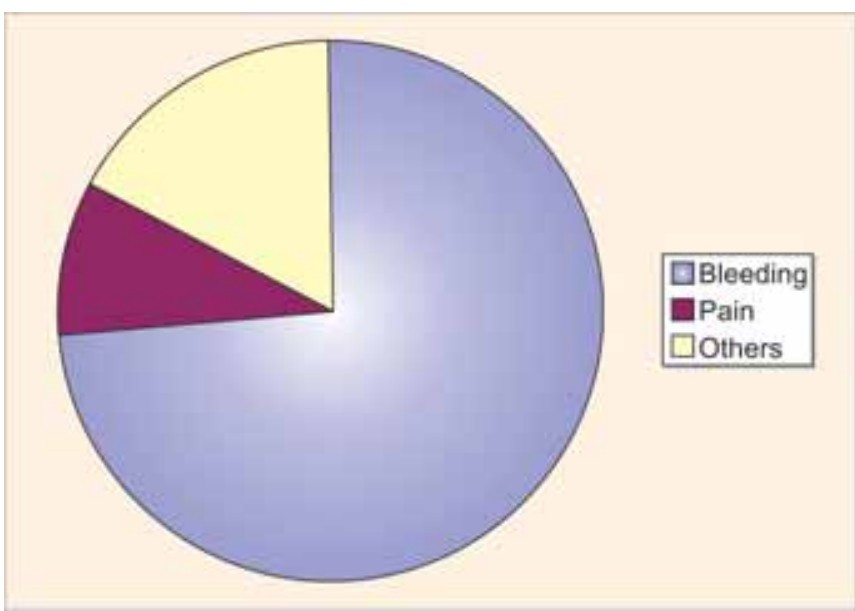

Fig. 3: The presenting complaint of patients at the EPAU

to surgery. M any women are often able to describe in detail very negative experiences surrounding miscarriage and they are often antagonistic to the medical and nursing staff involved in their care. ${ }^{6}$

In response to the dissatisfaction expressed by women about their care, there has been a rise in the number of hospitals offering an EPAU.
This is not an isolated situation in obstetrics as there has been amovetoward day carein many specialties. ${ }^{7,8}$ Thepractice appears to reduce costs and maximizes the use of resources. Day care management of common conditionsin obstetrics and gynecology has al so increased, e.g. col poscopy and outpatient hysteroscopy. ${ }^{9}$

The majority of patients can be assessed, monitored and treated without admission to hospital and this is the goal of an EPAU. Other authors noted that the early pregnancy assessment unit improved the qual ity of care and also produced considerable savings in financial and staff resources. ${ }^{10,11}$

W ith the development of urine and serum testing, pregnancy could be confirmed from as early as a missed period. It was not until the late 1970s that real-time ultrasound and transvaginal probes allowed accurate diagnosis in early pregnancy. Transvaginal ultrasound can visualize an intrauterine sac when the serum beta-hCG level reaches $750 \mathrm{IU} / \mathrm{l}-$ around five weeks' gestation. Fetal heart pulsation can be seen by six weeks, although identification with an abdominal transducer will be on average one week later.

Women, who were unwell, bleeding heavily or who had questionable adnexae with a positive pregnancy test (pregnancy of unknown location) were admitted through the usual channels and not asked to wait for an appointment on the unit. There will al ways be a proportion of women who are frightened by the loss or who are socially or geographically isolated and preferred admission.

The nationality of clients reflected the general population currently living in the country. The source of referral was mainly the emergency department which is in line with the policy of the unit whereby self-referrals have to be seen first at the emergency (Fig. 3). This practice multiplies the workload and it may be time to review it. Changing this to self-referral system would reduce a large volume of work at the emergency department.

When looking at the administration of the unit, we may note that when reviewing a year long practice in 2009, there were peaks and troughs periods as described in the figure (Fig. 5). This needs to be taken in consideration when organizing staffing. A uthors around the world help in management of such a unit by publishing their experiences. ${ }^{12}$

Bleeding, which in the majority of cases meant spotting, was the main reason for presenting. The majority had a second more powerful reason of needing reassurance (Figs 6 and 7). ${ }^{13}$ Here, it is necessary to point out that in our population, a huge emphasis is put on reproduction. This is reflected in that 90\% of these patients knew their exact last menstrual period and only $7 \%$ have ever used contraception.

We strongly believe that the establishment of the EPAU has positively affected the practice in our hospital. Other units noted that there is a strong demand and need for this service for couples who experience a miscarriage. ${ }^{14}$ To prove this, we compared admissions to the hospital during the year prior to its start and the year after. We must note that the hospital deliveries have increased from 14000 in 2007 to 15000 in 2009 (Table 1). 


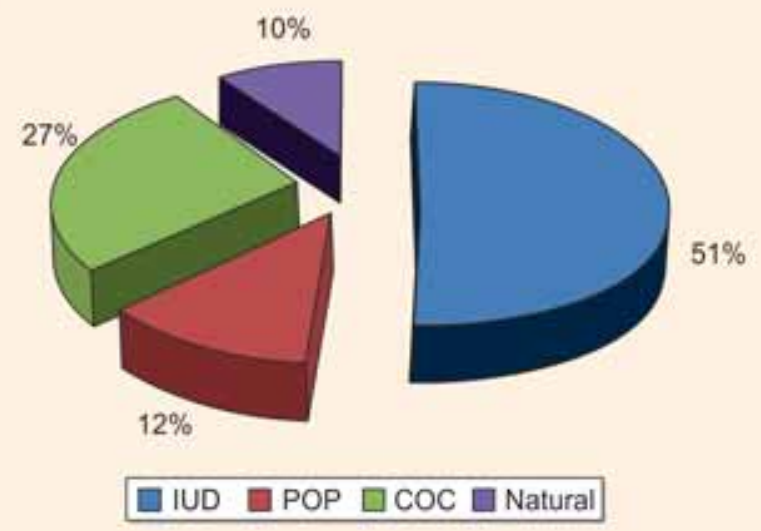

Fig. 4: The types of contraception ever used in the patients attending at the EPAU

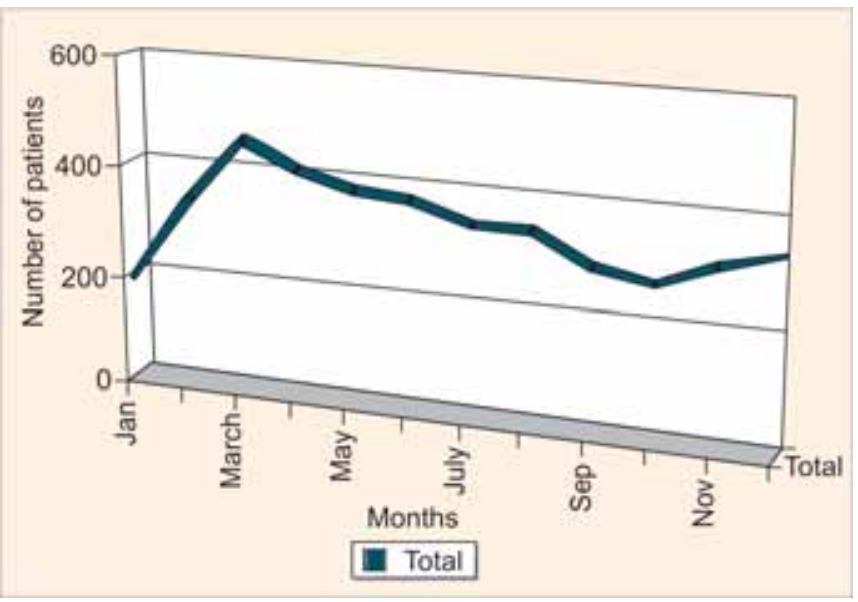

Fig. 5: The total number of visits to the EPAU in 2009

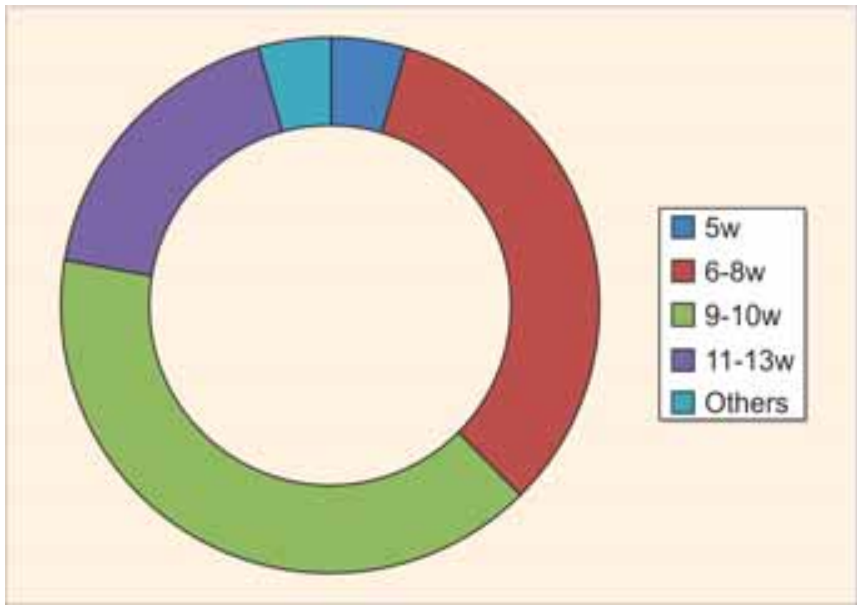

Fig. 6: The gestational age at presentation to the EPAU

We noted that the unit has almost halved the admissions for threatened miscarriage while reducing by one-third admissions in cases of ectopic pregnancy, having supported the practice of noninterventional management. This is in line with the findings of $\mathrm{HoO}$ et al. ${ }^{15}$

$M$ anagement of missed abortion al so benefits from having patients managed in an EPAU. ${ }^{16}$

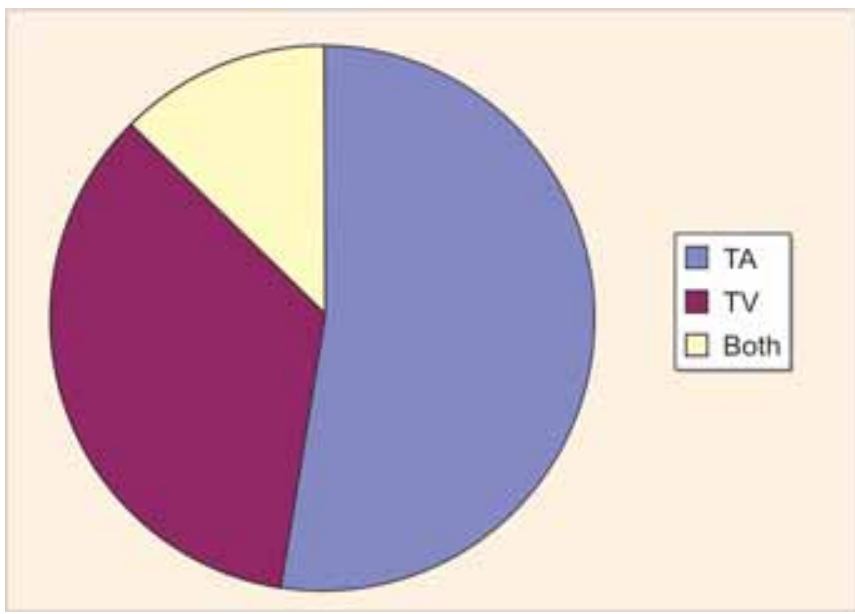

Fig. 7: The type of ultrasound performed on the patients during the study period at the EPAU

Table 1: A comparison of hospital admissions in the years 2007 and 2009, due to early pregnancy complications, showing the significant decrease in hospital admissions

\begin{tabular}{llll}
\hline Diagnoses & $\begin{array}{l}\text { \% of total } \\
\text { deliveries } \\
\text { in 2007 }\end{array}$ & $\begin{array}{l}\text { \% of total } \\
\text { deliveries } \\
\text { in 2009 }\end{array}$ & Change (\%) \\
\hline Missed & 5.06 & 3.66 & $<28$ \\
Ectopic & 2.14 & 1.88 & $<12$ \\
Spontaneous misc & 6.81 & 6.61 & $<3$ \\
Threatened misc & 1.45 & 0.84 & $<43$
\end{tabular}

The patient number is increasing which may necessitate running the clinic 7 days a week. The staff particularly the nursing have changed a number of times which each time was to the detriment of the service as it reduced the continuity of care for follow-up patients. The referrals are mainly from the emergency department which needs to change to self-referral to hel $p$ reduce unnecessary visits to the emergency department.

\section{CONCLUSION}

A significant proportion of women experience bleeding in early pregnancy resulting in considerable distress. They deserve access to a system where they can find out rapidly whether the pregnancy is continuing. One way of achieving this is by the introduction of EPAUs, so that they are cared for by specialist staff who aim to provide optimum care for any individual woman and her family. Such a system is attractive to managers as there is no great cost implication in comparison to the benefits and cost reduction due to reduced admissions.

The new service has clearly positively affected the provision of service at our hospital and we would recommend any busy hospital having such a unit.

\section{ACKNOWLEDGMENT}

The authors wish to acknowledge the colossal effort of both Professor A sim K urjak and Professor B adreldeen A hmed, the 
leaders of the fell owship program in ultrasound at D oha, Q atar which facilitated the beginning of this practice.

\section{REFERENCES}

1. Regan $L, B$ aude PR, T rembath PL. Influence of past reproductive performance on risk of spontaneous abortion. Br M ed J 1989;299:541-45.

2. Cuisinier MC, Kuijpers JC, Hoogduin CA, de Graauw $C P$, J anssen $\mathrm{HJ}$. M iscarriage and stillbirth: Time since the loss, grief intensity and satisfaction with care. Eur J Obstet Gynecol Reprod Biol 1993;52:163-68.

3. Welch ID. M iscarriage, stillbirth or newborn death: Starting a heal thy grieving process. Neonatal Network 1991;9:53-57.

4. Royal College of Obstetricians and Gynaecologists (RCOG). The management of early pregnancy loss. L ondon (UK ): R oyal College of Obstetricians and Gynaecologists (RCOG); Oct 2006;18(Green-top guideline; no. 25).

5. Royal College of Obstetricians and Gynaecologists and Royal College of Radiologists J oint Standing Committee. Guidance on UItrasound Procedures in Early Pregnancy. London: RCOG 1995.

6. Cormell M. Just another miscarriage? Nurs Times 1992, 1998;88:41-43.

7. Bartoni C, Zurrida S, Clemente C, Cascinelli N. Outpatient surgical treatment of cutaneous melanoma. M elanoma Res 1992;1:385-90.
8. Scott Giebink G. Care of the ill child in day-care settings pediatrics. 1993;91:229-33.

9. Nagele $F, O^{\prime}$ Connor $H$, Davies A, Badeery A, M ohamed $H$, Magos A . 2500 outpatient diagnostic hysteroscopies. Obstet Gynecol 1996;88:87-92.

10. Bigrigg M A, R ead M D. M anagement of women referred to an early pregnancy assessment unit: Care and cost effectiveness. BMJ 1991;302:577-79.

11. Davies M, Geoghegan J. Developing an early pregnancy assessment unit. N urs Times 1994;90:36-38.

12. Ultrasound and early pregnancy. By N icole S. W inkler and A nne K ennedy Print Publication Y ear. 2010. Online Publication Date: October 2010;39-52.

13. Bottomley C, Van Belle V, M ukri F, K irk E, Van Huffel S, Timmerman $D, B$ ourne $T$. The optimal timing of an ultrasound scan to assess the viability of an early pregnancy. UItrasound Obstets Gynecol 2009;24:1811-17.

14. Turner M J, Flannelly G M , W ingfield M , et al. The miscarriage clinic: A $n$ audit of the first year. $\mathrm{Br} J$ Obstet Gynaecol 1991;98:306-08.

15. W Hoo, et al. Reducing the follow-up required in women with a failing pregnancy of unknown location. Ultrasound in Obstetrics and Gynaecology. M onday 11 October 2010.

16. El-R efaey $\mathrm{H}, \mathrm{H}$ inshaw $\mathrm{K}$, Henshaw R, Smith $\mathrm{N}$, Templeton $\mathrm{A}$. $M$ edical management of missed abortion and anembryonic pregnancy. B M J 1992;305:1399. 